\title{
Motion Belts: Visualization of Human Motion Data on a Timeline
}

\author{
Hiroshi YASUDA ${ }^{\dagger a)}$, Student Member, Ryota KAIHARA ${ }^{\dagger}$, Nonmember, $_{\text {Suguru }}$ SAITO $^{\dagger}$, \\ and Masayuki NAKAJIMA ${ }^{\dagger}$, Members
}

SUMMARY Because motion capture system enabled us to capture a number of human motions, the demand for a method to easily browse the captured motion database has been increasing. In this paper, we propose a method to generate simple visual outlines of motion clips, for the purpose of efficient motion data browsing. Our method unfolds a motion clip into a 2D stripe of keyframes along a timeline that is based on semantic keyframe extraction and the best view point selection for each keyframes. With our visualization, timing and order of actions in the motions are clearly visible and the contents of multiple motions are easily comparable. In addition, because our method is applicable for a wide variety of motions, it can generate outlines for a large amount of motions fully automatically.

key words: computer animation, motion capture, data visualization

\section{Introduction}

As motion capture system enables us to take human motions into a computer, a demand for the method to easily find a target motion from a large amount of captured motions is increasing. This aim mainly involves two distinct issues: retrieval and browsing. In this paper, we propose a method to visualize the outlines of motion clips for the purpose of efficient browsing. For efficient browsing, the visualized results should be clear for a wide variety of motions, and easily comparable for the contents of multiple motions. We will show that our proposed method works well in both aspects.

Currently, video clip is widely used to visualize motion data. Because it visualizes motion in a way we normally observe human motions, the contents of the motion are easily understood when only a single motion is concerned. However, for browsing multiple motions, it is not only time consuming but also hard to compare the contents, especially when temporal differences are involved; for instance, when similar motions occur at different timings. The problem becomes more important when it is an online database that needs extra time for downloading the video files.

Showing static images of keyframes with some semantic signs is the better alternative for browsing multiple motions. Because the outline of the motions are illustrated as static images, it is easier to browse and compare multiple motions regardless of their temporal differences. However, as a trade-off, temporal information including the timing and the order of actions is not preserved. Moreover, with exist-

Manuscript received October 2, 2007.

${ }^{\dagger}$ The authors are with the Graduate School of Information Science \& Engineering, Tokyo Institute of Technology, Tokyo, 152 8552 Japan.

a)E-mail: yasuda@img.cs.titech.ac.jp

DOI: $10.1093 /$ ietisy/e $91-$ d. 4.1159

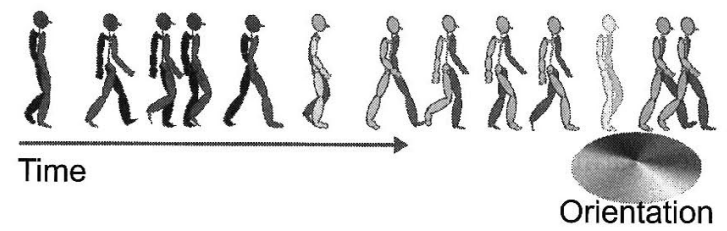

Fig. 1 Motion belt of walking with a 90 degree turn; keyframe color indicates the hip orientation.

ing methods, the content of the motion is barely comprehensible when the motion has small spacial locomotion because of the occlusions between keyframes placed in a 3D space. Thus, the methods need manual adjustments to generate clear visualizations and therefore they are not directly applicable for a large number of data.

We therefore propose a novel visualization method that unfolds the extracted keyframes on a timeline as shown in Fig. 1. We call this motion belt. In a motion belt, even for the motions with small locomotion, the keyframes are placed with intervals according to their time intervals. Furthermore, the timings and the temporal orders of actions are preserved and clearly visible. Thus, our method provides better understanding for a wide variety of motions, as well as easy browsing and comparison for multiple motions.

\subsection{Overview and Contributions}

Our method consists of three steps. First, we extract semantic keyframes in a motion, then we select the best view direction for each keyframe, and finally we visualize the projected keyframes on a timeline.

The key contributions of this work are as follows:

- Our method is able to automatically generate visual outlines for a wide variety of motions.

- Motion belts provide ways to easily compare and browse multiple motions.

- The timing and order of the events in the motion clips as well as their spatial informations are clearly visualized by motion belts.

- Our method extracts semantic keyframes and displays the frames with the best view direction that reflects the motion contents.

We start by reviewing related works on motion database and motion visualization in Sect. 2. The keyframe extraction method is then described in Sect. 3. In Sect. 4, 
we explain the method that finds the best view direction to project the extracted keyframes. Final visualization results of various types of motion are presented in Sect. 5. In Sect. 6, we summarize the achievements and discuss future works.

\section{Related Works}

One of the popular topics of motion database is motion data retrieval. Same as other media retrieval researches, distance measurement, retrieval accuracy and search speed are the primal issues. Dynamic programming (DP) based measurements are widely used to compare motion clips, because it is applicable for the motions that have diverse lengths. However, comparing long sequence with DP is time consuming. There are some attempts to speed up the process by limiting matching to subsequence [1], [2]. In order to improve the retrieval quality, some researches set their aim on defining a similarity measure that captures the high level semantic concepts [3]-[5]. In our work, we take advantage of the similar features used for the semantic encoding of motion [4], [5] in order to extract semantic keyframes.

Motion data browsing is another topic for motion database. Sakamoto and Kuriyama [6] proposed a searching interface that enables the user to explore the posture space of the motions in the database. The posture images are distributed on a $2 \mathrm{D}$ plane while preserving the similarity between them. Therefore, users can easily find a posture that is used as a searching query. Slyper and McCann extended the idea to explore motion data directly by showing video clips instead of posture images [7].

Visual outlines of motions provide more basic way to explore the database. The outlines of motions are illustrated as the images of static keyframes and some semantic annotations [8]-[10]. While existing outline methods focus on visualizing only spacial variation of the motions, our approach visualizes also temporal information by adding the concept of timeline. Therefore, our method provides clearer visualization for a wide variety of motions.

\section{Keyframe Extraction}

Keyframe extraction has two steps; First encode every posture of motion into a set of feature values, then extract the frames that have distinct changes of the values as keyframes.

To detect the frames that have semantic meanings such as contacts between objects and body parts, up and down of arms and back and forth of limbs, we adopt distance based features in contrast to the existing keyframe extraction methods [1], [8], [9]. For motion retrieval purpose, Müller et al. showed that the distance based features are suitable to describe a motion as series of semantic status [5]. Therefore, we use 13 distances between body parts extending their feature sets for keyframe extraction: 7 distances between the head, hands and feet (Fig. 2, D1-D7), 4 distances of hands and feet from the frontal plane that divides front part of the body and rear part of the body (D12-D15), and 2 distances

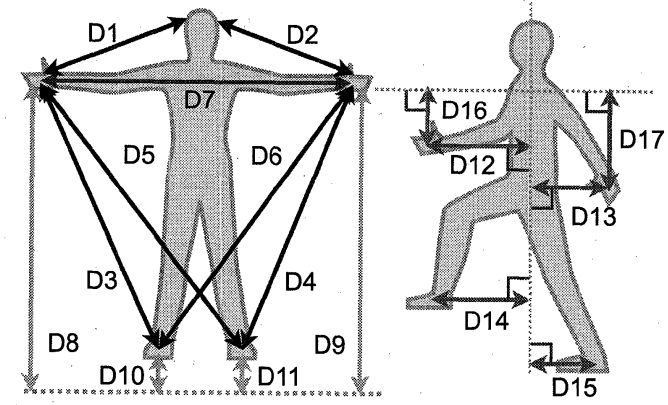

Fig. 2 Distances used to encode a posture.

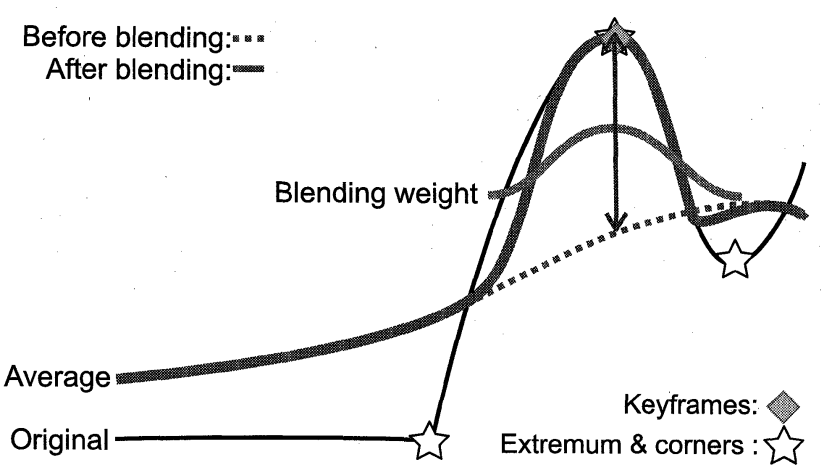

Fig.3 Average curve updating.

of hands from the shoulder height plane (D16, D17). In addition, to take into account events such as the contact with objects in the environment, we propose to include hands and feet heights from the floor (D8-D11). Thus, in total, a posture is encoded with a set of 17 values as illustrated in Fig. 2. The dotted lines in the figure indicate the floor, shoulder height and the frontal plane.

Now, a motion can be described as a high dimensional function of 17 time varying values. Keyframes in the data can be considered now as distinct changes of the curve. In order to extract the keyframes, we propose a method that improves over the method proposed by Assa et al. [8], where they extracts local extremum of the curve as keyframes.

Here, we explain their algorithm briefly. First, they calculate an average curve by smoothing the original curve using a Gaussian kernel. Next, the frame that has the maximum distance between these two curves is marked as a keyframe. Then, the average curve is updated by blending with the original curve as shown in Fig. 3. A fixed size Gaussian kernel with isotropic diffusion is used as a weighting function for the blending. This process is repeated iteratively until sufficient number of keyframes are extracted or the maximum distance between the curves become under a threshold. Thus, this method can extracts extremum (keyframes) with the order that reflects the magnitude of the difference from the local average. Therefore, by simply taking the necessary number of keyframes from the beginning the information given by displayed keyframes is maximized. This characteristic has large benefits for motion belts. It allows to change the number of keyframes easily according to 


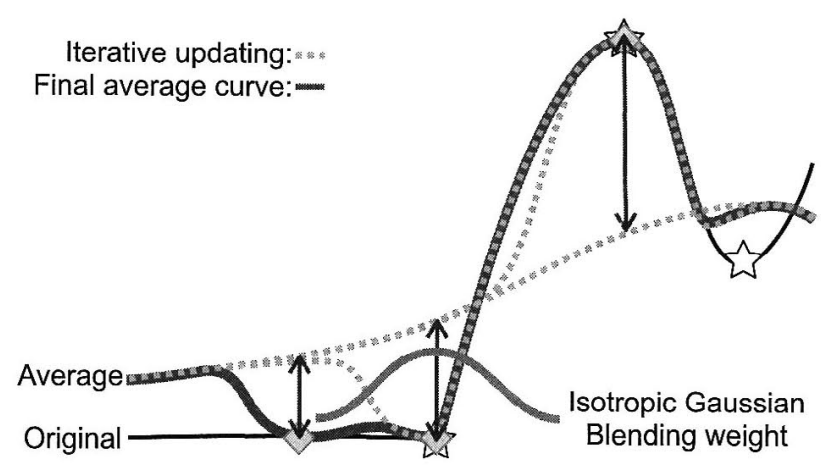

Fig. 4 Artifacts caused by isotropic Gaussian weighting.

its timeline scale.

However, the extremum extraction method is not directly applicable to the curve of our feature values, because the curve usually has step edges at contact events. Therefore, not only extremum but also the corners should be considered as keyframes. As illustrated in Fig. 4, when an isotropic Gaussian is used for updating the average curve, an undesirable keyframe is sampled where the curve has only subtle changes while an extremum right side is left out.

In order to reduce this artifact, we propose to use a skewed Gaussian kernel instead of the isotropic Gaussian kernel as a weighting function. The shape of the kernels are automatically adjusted to give low sampling density in a direction where the original curve does not change largely. The shape adjusting process is as follows.

The process is performed independently for the frames before the keyframe and after it. For a simple explanation, we consider one feature value case in the following, while we actually use 17 dimensional vectors.

First, we calculate the local difference $y$ between the average curve $\bar{C}$ and the original curve $C$ around the keyframe $k: y_{i}=\|C(i)-\bar{C}(i)\| /\|C(k)-\bar{C}(k)\|$. Where, $i$ is the neighbor frames of the keyframe $k$. In our experiments, 40 neighbors of the keyframe are used for each direction. We denote by $y_{i}$ the normalized residual errors. By adjusting the blending weight proportional to the residual errors, the average curve will be updated to fit the original function locally as shown in Fig. 5. Then, the artifact around the corner edges will be reduced. We start by finding a parameter $\sigma$ of the Gaussian function, $\exp \left(-x^{2} / \sigma^{2}\right)$, that best fits the residual error distribution, minimizing the squared error $E$ :

$$
E=\sum_{i}\left(y_{i}-\exp \left(-\frac{x_{i}^{2}}{\sigma^{2}}\right)\right)^{2},
$$

where $x_{i}=\|k-i\|$. This minimization problem can be transformed into a linear regression problem that minimizes $J$ :

$$
J=(X-\sigma Z)^{T}(X-\sigma Z), 0<y_{i} \leq 1,
$$

where $X$ is the column vector of $x_{i}$, and $Z$ is the column vector of $\sqrt{-\log y_{i}}$. Note that $y_{k}=1$ and $y_{i}$ of neighbor frames take values between 0 to 1 , and the neighbors with $y_{i}=0$ are excluded for the fitting. We also constrain $\sigma$ from

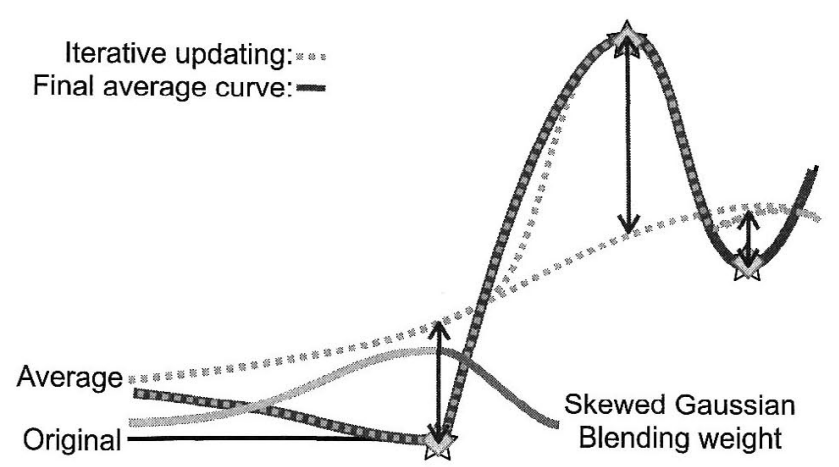

Fig.5 Blending with skewed Gaussian weighting.

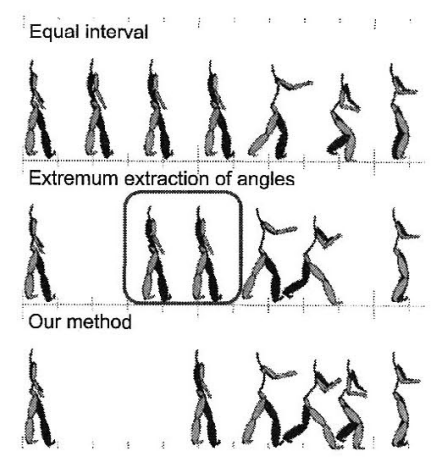

Fig. 6 Extracted keyframes for a catch ball motion.

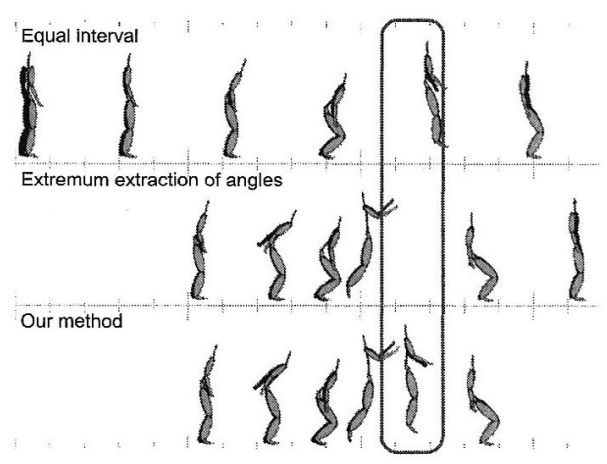

Fig. 7 Extracted keyframes for a jumping motion.

20 to 200 for stability. Finally, the size $\gamma$ of the weighting Gaussian function, $\exp \left(-x^{2} / \gamma^{2}\right)$, is decided proportionally to $\sigma$; in our experiments $\gamma=2 \sigma$ worked well.

Figures 6 and 7 show some keyframe extraction results. In order to show the effectiveness of our method, we compare the results with:

Regularly spaced keyframes : where the keyframes are sampled with equal interval.

Keyframes as the angular value extremum : where the keyframes are sampled by Assa's method [8] with angular based feature values.

The catch ball motion includes both static and brisk durations. While the extremum extraction gives redundant keyframe when the motion switches from static to brisk, our 
method extracts keyframes that reflects the intensity of the motion. For the jumping motion result, our method successfully captures the moment at top of jumping, because of the distances from floor we introduced.

For motion belts, when the motion data is rather static, there will be large blanks on a timeline between keyframes. Therefore, in order to show that there is no distinct event in these duration, we fill the blanks by showing tinted frames, located at even intervals according to the timeline scale.

\section{View Point Selection}

Because the extracted keyframes have a 3D structure, we need to decide view points to project each keyframe into a 2D plane. Since it is hard to recognize the whole contents of a motion clip with a single view point, we determine the projection for each keyframe using local information.

One of the main difficulties to determine the best view point is the high dimensionality of the camera parameter space. In the case of a pinhole camera, there are 7 parameters to describe one view point [11]: 3 for the target position, 3 for the camera rotation and one for the focal distance as shown in Fig. 8. Therefore, we start by reducing the number of the camera parameters. First, in order to locate a keyframe image at the center of its projection plane, the target position of the camera is set at the vertical of the root joint's position of the keyframe. The height of the target position is fixed for all keyframes to preserve the height information in the projected image. We also choose to use parallel projections without zooming in order to get a uniform scale of the human figure along the movement. Therefore, we can discard the focal distance.

To reduce further the camera parameters, we take advantage of the experimental knowledge in cognitive science. Dittrich [12] showed that motions are recognized much better and faster when presented in the normal orientation rather than upside down. Furthermore, Daems and Verfaillie [13] indicate that the identification performance of human actions increases when the action had been observed in the orientation before. Supported by these knowledge, we set the roll rotation to preserve gravity direction, and fixed the tilt rotation to zero to be a sideways projection which is the natural way of observing human motion. Figure 9 shows the final camera setting for our keyframe projection.

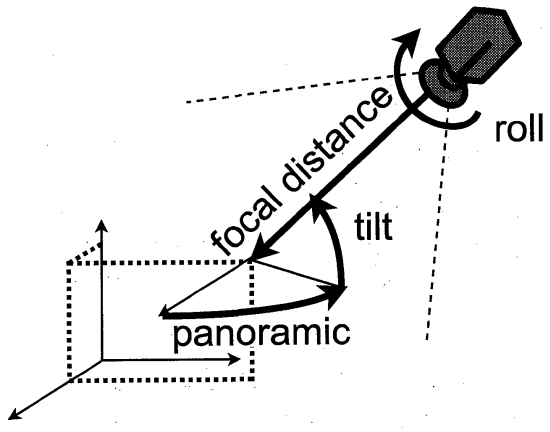

Fig. 8 Pinhole camera parameters [11].
Now we only have one parameter left, panoramic rotation. In order to decide the rotation, we need a strategy to measure the goodness of the view direction. For static $3 \mathrm{D}$ objects case, the view goodness is defined based on the object's geometry features [14]-[17]. However, for motion data, the body geometry changes according to the character to which the motion is attached to. On the other hand, it is known that just showing joint positions by dots is enough for human to recognize not just the action but also the gender and emotion of the actor [18]. Therefore, we define the best view direction as the view direction that gives the best visibility of the differences and the trajectories of joint positions. In addition, because we decide the view direction for each keyframe, coherency is also important factor for better understanding of motion contents.

We first consider to capture characteristics of a single posture, then we define a feature that includes trajectories and coherency. The feature of a posture at a current moment is defined as differences of joint positions from neutral standing posture as illustrated in Fig. 10. The vectors of the differences are then gathered at the root joint's position and projected into a horizontal plane, because the vertical components of the vectors are constant for arbitrary panoramic rotation. We call the projected vectors difference vectors.

Next, in order to take into account the trajectories, we also calculate the difference vectors for neighbor frames. This process also introduces coherency between view directions for neighbor keyframes. In our experiments, we could get stable results with 50 neighbors that are resampled at 6 frames/sec for this process. Finally, we simply use PCA to find the view direction that maximizes the distribution of the end tips of the difference vectors as shown in Fig. 11.

Because we project 3D motion data into 2D plane, we cannot avoid the lose of some information such as global

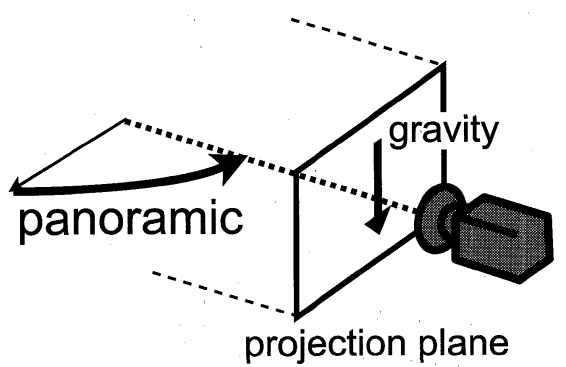

Fig.9 Camera setting for the keyframe projection.

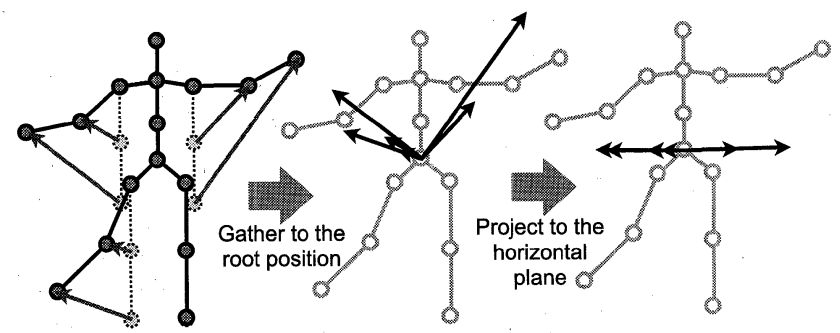

Fig. 10 Pose feature. 


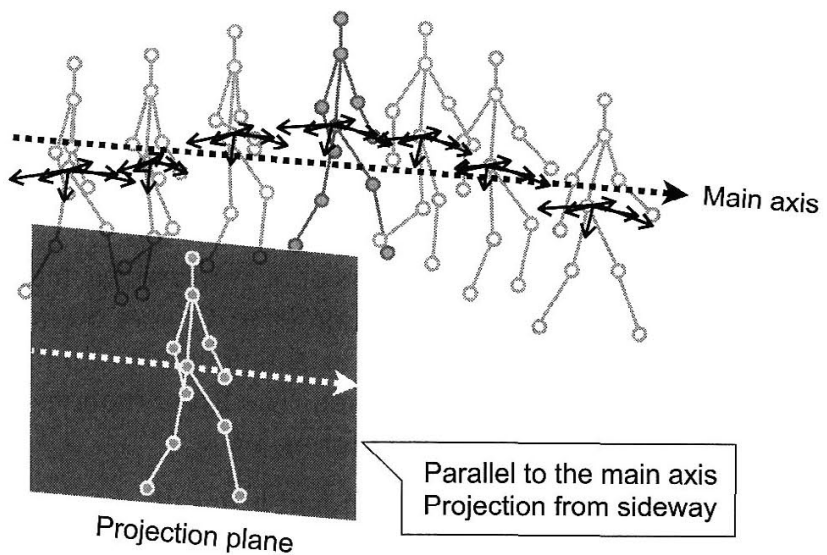

Fig. 11 Projection selection algorithm.

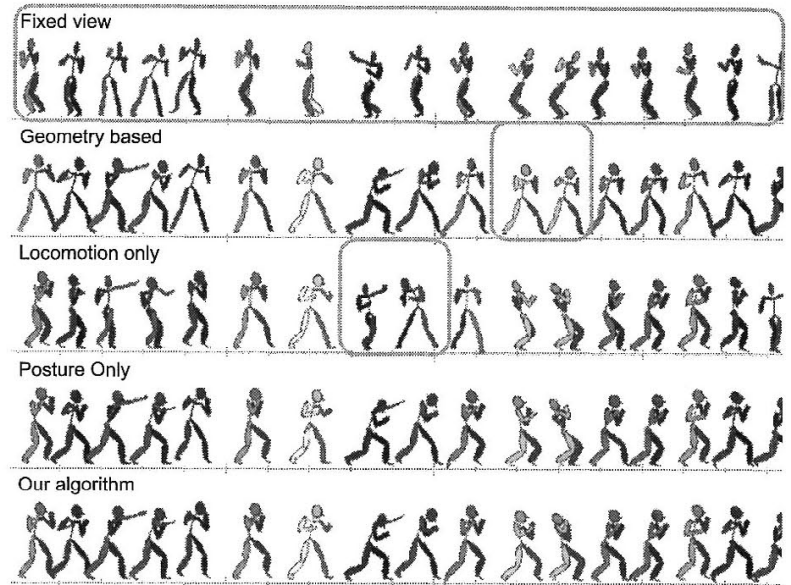

Fig. 12 Boxing.

transition in our case. To preserve as much information, we propose to use color to indicate the global orientation of the hip as shown in Fig. 1.

Figures 12, 13, 14 and 15 show the projection results of boxing, picking up, jumping and walking with a $90^{\circ}$ turn. As explained above, our method uses non-geometry features based on a posture and trajectories of joint positions and locomotion. Therefore, In order to show the effectiveness of our methods, we compare with the following methods.

Geometry based feature: Joint positions without subtracting standing posture,

Locomotion only: Root joint trajectory,

Posture only: Difference vectors of single frame only,

Fixed view point is also shown as a reference.

With geometry based features, the view points are unstable when the body geometry changes frequently like in the walking result. For jumping and boxing, it does not reflect well the motion contents, because the influence of the shoulder width is larger than the trajectories. Locomotion only feature gives unstable projections when the motion does not have large locomotion: boxing and picking up. Posture only feature gives reasonable projection for each

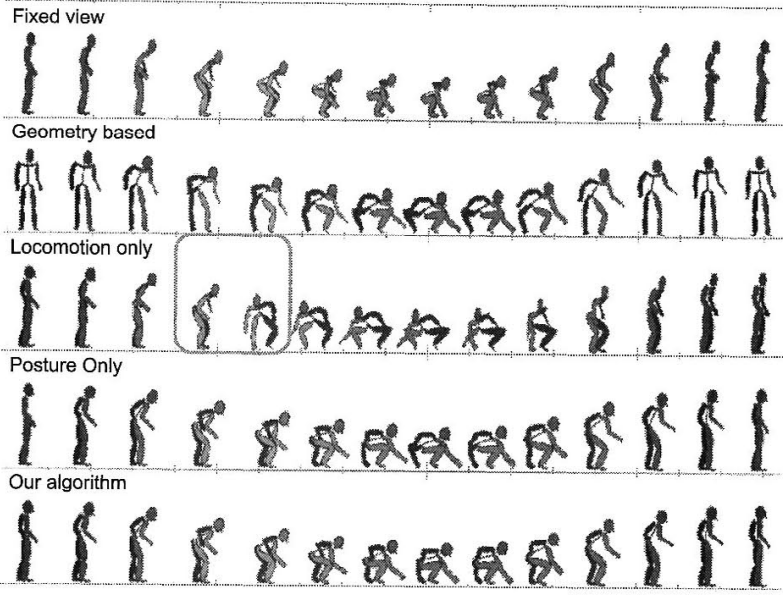

Fig. 13 Picking up.

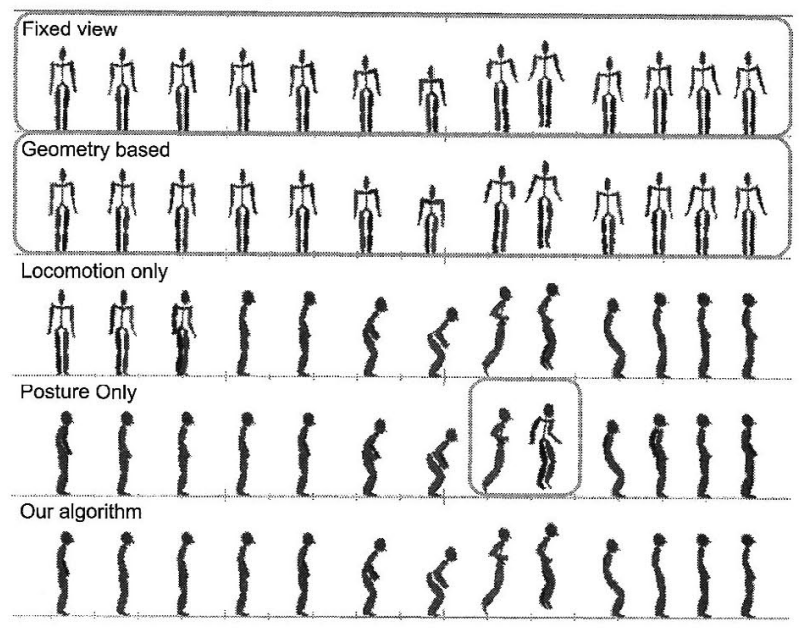

Fig. 14 Jumping.

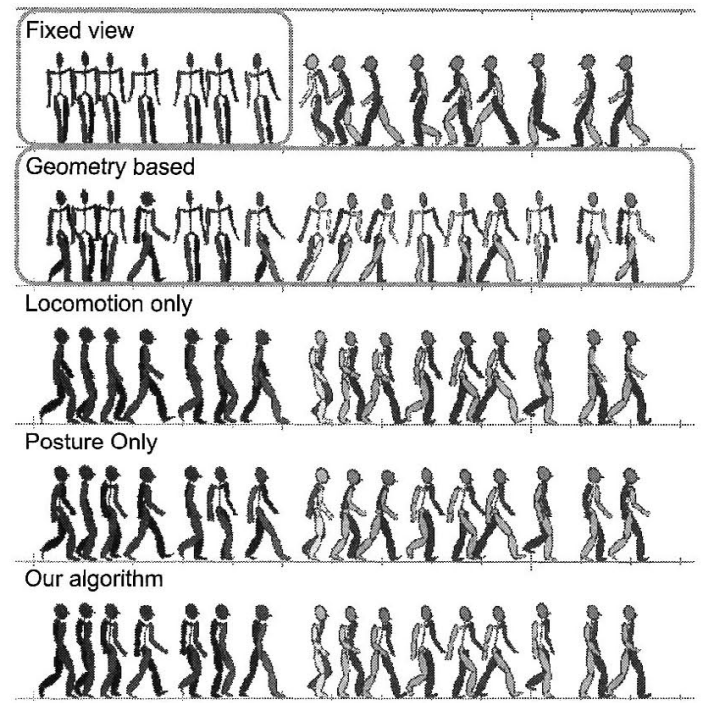

Fig. 15 Walking with 90 degree turn.

keyframes, but still lacks coherency in the result of jumping, when the pose feature conflicts with trajectory features. Proposed algorithm select reasonable and stable projections for 
all examples.

\section{Results and Applications}

We applied the proposed method to the motion data in CMU motion database [19]. Figure 19 shows instances for variety of motions including the motion without large locomotion. Motion belts successfully visualize the motion contents, regardless of the magnitude of the spacial locomotion. Furthermore, the order of the actions and the differences of motion length are clearly visualized. Global rotations are also clearly visible by the rainbow like color changing according to the hip orientation.

One of the straight forward applications of motion belts is to generate image thumbnails for motion database. In the case of the CMU motion database [19], a list of content descriptions and video clips are used to preview the contents of motions. However, video clips are time consuming to browse large amount of data. In addition, although the literal descriptions help to quickly find a group of motions that includes the motion the user is looking for, they do not have enough details to compare similar motions because they do not show the motion itself. Motion belts can assist the user to recognize how the motions are actually performed, cooperating with the content descriptions. Figure 18 shows

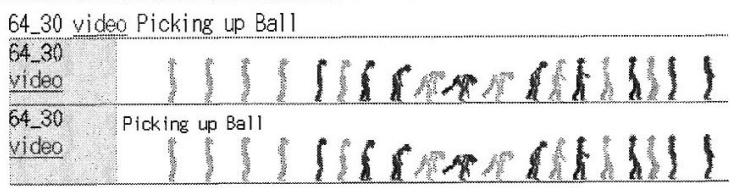

Fig. 16 List forms $\mathrm{CD}, \mathrm{MB}$ and $\mathrm{CD}+\mathrm{MB}$ from top to buttom. instances of motion belts that have the same content description "soccer-kick ball" in the database. All the motions contain a single ball-kicking action but at different timings. The timing differences are clearly visualized in the motion belts, therefore, user can easily compare the contents and find the target motion quickly without checking video clips.

In order to see the effectiveness of our method, we measured the time to find a given motion from a list of $50 \mathrm{mo}-$ tions. The target motion is given as an animation clip that allows to control view point and time. The list of motions is displayed in one of the following forms.

CD: Data ID, content description and a link to the video clip.

MB: Data ID, motion belt and a link to the video clip.

CD+MB: Data ID, content description, motion belt and a link to the video clip.

Examples are shown in Fig. 16. For all forms of the list, the

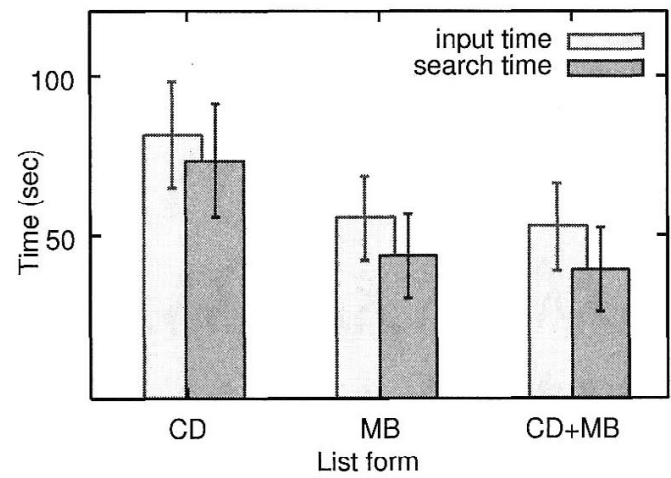

Fig.17 Time for searching a motion from 50 motions.

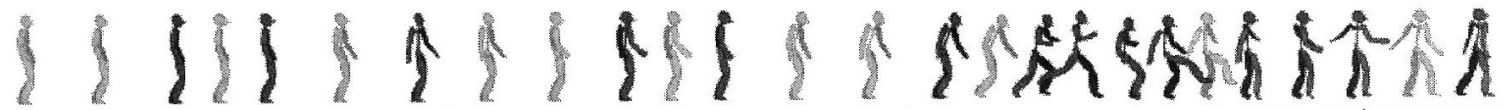 M1918}


YASUDA et al.: MOTION BELTS: VISUALIZATION OF HUMAN MOTION DATA ON A TIMELINE

1165

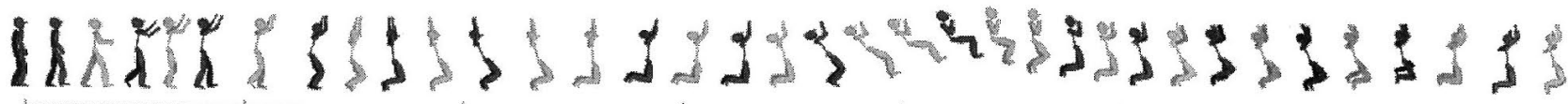
lis

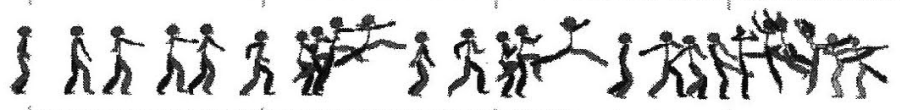

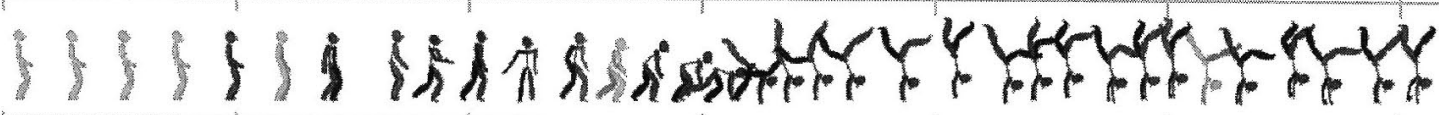

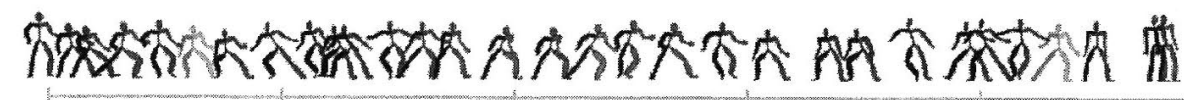

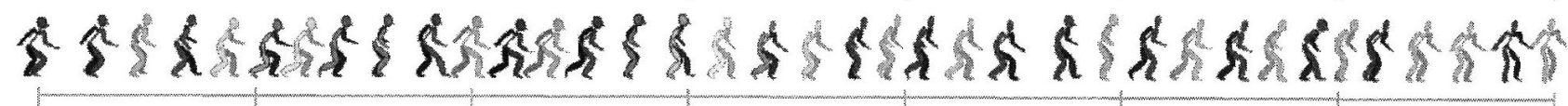

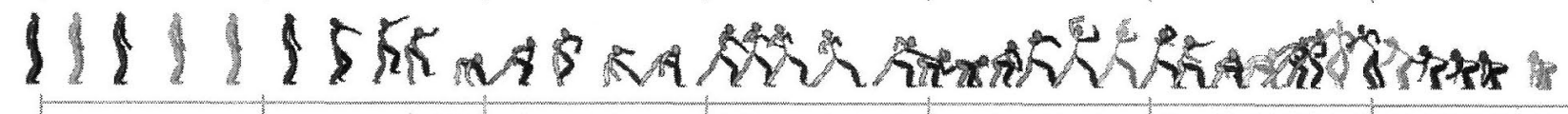

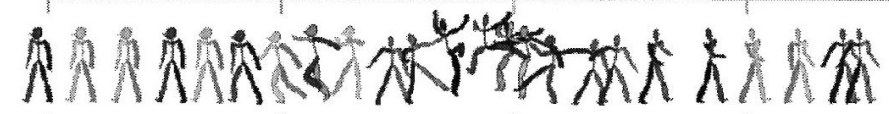

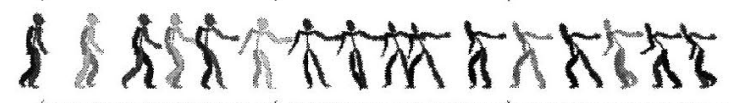

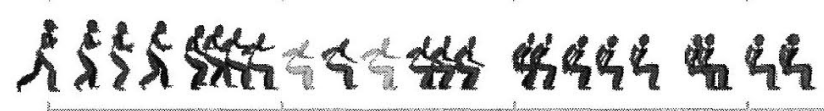

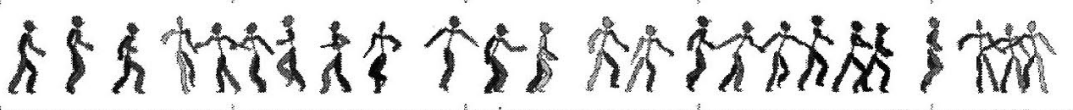

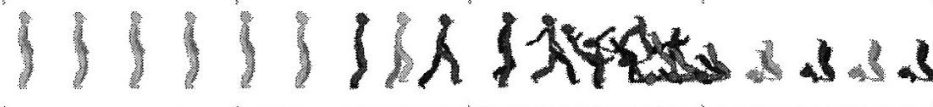

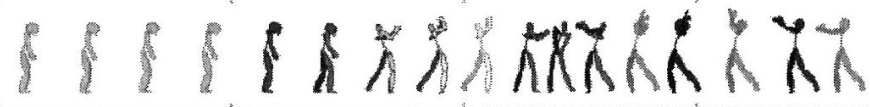

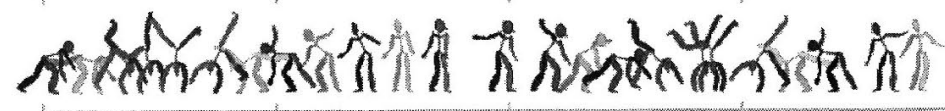

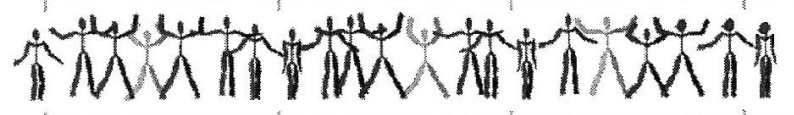

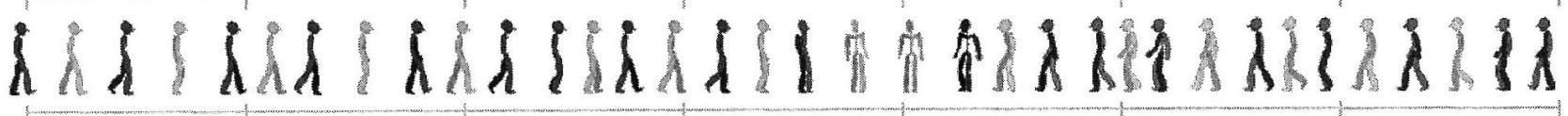

Fig. 19 Motion belt examples: pulling up, laughing, dancing, standing on hands, side stepping, sneaking, monkey (mime), jumping kick, pulling, sitting on a chair, skipping round,
slipping down, golf swinging, cartwheels, jumping jacks and walking with a 180 degree turn.

elements are sorted according to their alphabetical order of the content descriptions. The form CD is widely used for motion databases including the CMU database we used for this experiment.

We use two monitors for displaying the target motion and the motion list separately so that the subjects can see 
the target motion anytime while searching on the list. The numbers of list items displayed on the screen at once for the forms $\mathrm{CD}, \mathrm{MB}$ and $\mathrm{CD}+\mathrm{MB}$ are 50, 24 and 20 respectively. A scroll bar is used when further exploration is necessary. We measured the time from when the target motion is displayed to when the correct data ID is entered. We call this input time. 8 subjects who do not familiar with the motion database are involved. The subjects include a native speaker of English. We measured mean input time for 90 trials in total containing 30 trials for each list form. The order of the list form is decided to be random. In each trial, 50 motions are randomly sampled from 2074 motions in the database. Before starting the searching trials, we let the subjects practice approximately 10 minutes to get used to the content descriptions and the motion belts.

In order to measure the time necessary for the operation of the experimental tool itself, we gave a task to scroll a list to the bottom and input a data ID using a list contains 50 instances of a single motion. For each subject, we measured the average time of the task repeated 10 times for each list form. Then, we calculate the time took for searching by subtracting the tool operation time from the input time. Figure 17 shows means and standard deviations of the input time and the searching time for each list form.

Although, the expression CD has advantage in the tool operation time since it does not need scrolling, it took the longest time in average for both input and search time. Although, the form $\mathrm{CD}+\mathrm{MB}$ took the shortest time for searching in average. It is approximately 1.9 time faster than the form $\mathrm{CD}$. On the other hand, the results of the form $\mathrm{MB}$ and the form $\mathrm{CD}+\mathrm{MB}$ does not have significant difference. This indicates, the subjects tends to take advantages of motion belts even when the content descriptions are shown. Thus, we confirmed that motion belts are effective for motion data browsing.

\section{Conclusion}

We proposed motion belts as a method to visualize motion data. It unfolds a motion into a 2D stripe of keyframes along a timeline. To achieve the goal, we proposed the method of semantic keyframe extraction based on distance features. We also proposed the method to project keyframes in a way that reflects the contents of the motion. We believe that this is the first attempt to determine good view direction for motion data. With motion belts, timing and order of the events in the motion clip are clearly visualized. We demonstrated that motion belts are effective for a wide variety of motions regardless of their magnitude of locomotion and temporal differences.

In the current view direction decision, we treat whole joints equally. However, user might want to put emphasis on particular body parts. In the case of hand gestures, for instance, the visibility of lower body could be ignored and zooming on the arms might give better understanding. Thus, controlling view parameters according to the user's region of interest can be a future work.
When a long motion that includes several types of actions is contracted to a short motion belt, it is hard to control keyframes without losing information for each actions.

Preprocessing the motion data with motion segmentation algorithms [20] can be a solution for this problem. With the segmentation, motion clips can be controlled to keep them short and to have a small variation of actions.

\section{References}

[1] F. Liu, Y. Zhuang, F. Wu, and Y. Pan, "3d motion retrieval with motion index tree," Computer Vision and Image Understanding, vol.92, no.2-3, pp.265-284, 2003.

[2] K. Forbes and E. Fiume, "An efficient search algorithm for motion data using weighted pca," SCA '05: Proc. 2005 ACM SIGGRAPH/Eurographics symposium on Computer animation, pp.6776, ACM Press, New York, NY, USA, 2005.

[3] L. Kovar and M. Gleicher, "Automated extraction and parameterization of motions in large data sets," ACM Trans. Graph., vol.23, no.3, pp.559-568, 2004.

[4] M. Müller, T. Röder, and M. Clausen, "Efficient content-based retrieval of motion capture data," ACM Trans. Graph., vol.24, no.3, pp.677-685, 2005.

[5] M. Müller and T. Röder, "Motion templates for automatic classification and retrieval of motion capture data," SCA '06: Proc. 2006 ACM SIGGRAPH/Eurographics Symposium on Computer Animation, pp.137-146, Eurographics Association, Aire-la-Ville, Switzerland, Switzerland, 2006.

[6] Y. Sakamoto, S. Kuriyama, and T. Kaneko, "Motion map: Imagebaséd retrieval and segmentation of motion data," SCA '04: Proc. 2004 ACM SIGGRAPH/Eurographics Symposium on Computer Animation, pp.259-266, ACM Press, New York, NY, USA, 2004.

[7] R. Slyper and J. McCann, "A hierarchical self-organizing map for motion exploration," 2007 ACM SIGGRAPH / Eurographics Symposium on Computer Animation Poster Proceedings, Aug. 2007.

[8] J. Assa, Y. Caspi, and D. Cohen-Or, "Action synopsis: Pose selection and illustration," ACM Trans. Graph., vol.24, no.3, pp.667-676, 2005.

[9] N. Shimuta and S. Kuriyama, "Summarization of character animation using image feautures," Tech. Rep. 91, IPSJ SIG Technical Report, Aug. 2006.

[10] S. Bouvier-Zappa, V. Ostromoukhov, and P. Poulin, "Motion cues for illustration of skeletal motion capture data," Non-Photorealistic Animation and Rendering 2007, pp.133-140, Aug. 2007.

[11] M. Christie and P. Olivier, "Camera control in computer graphics," Eurographics, vol.25, no.3, pp.89-113, 2006.

[12] W.H. Dittrich, "Action categories and the perception of biological motion," Perception, vol.22, no.1, pp.15-22, 1993.

[13] A. Daems and K. Verfaillie, "Viewpoint-dependent priming effects in the perception of human actions and body postures," Visual Cognition, vol.6, pp.665-693, 1999.

[14] T. Kamada and S. Kawai, "A simple method for computing general position in displaying three-dimensional objects," Comput. Vis. Graph. Image Process., vol.41, no.1, pp.43-56, 1988.

[15] P.P. Vazquez, M. Feixas, M. Sbert, and W. Heidrich, "Viewpoint selection using viewpoint entropy," Proc. Vision, Modeling, and Visualization (VMV) Conference, pp.273-280, Nov. 2001.

[16] P.P. Vazquez, M. Feixas, M. Sbert, and A. Llobet, "Viewpoint entropy: A new tool for obtaining good views of molecules," VISSYM '02: Proc. Symposium on Data Visualisation 2002, pp.183188, Eurographics Association, Aire-la-Ville, Switzerland, Switzerland, 2002.

[17] C.H. Lee, A. Varshney, and D.W. Jacobs, "Mesh saliency," ACM Trans. Graph., vol.24, no.3, pp.659-666, 2005.

[18] G. Johansson, "Visual perception of biological motion and a model 
for its analysis," Perception and Psychophysics, vol.14, pp.201-211, 1973.

[19] CMU graphics lab motion capture database, http://mocap.cs.cmu.edu/

[20] J. Barbic, A. Safonova, J.Y. Pan, C. Faloutsos, J.K. Hodgins, and N.S. Pollard, "Segmenting motion capture data into distinct behaviors," Proc. Graphics Interface, pp.185-194, May 2004.

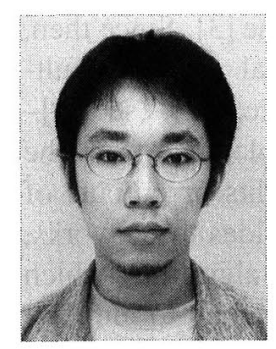

Hiroshi Yasuda was born in Kagawa, Japan on January 25, 1980. He received the B.S degree from Tokyo University of Science in 2003. Since April 2003, he has been in Graduate School of Information Science and Engineering, Tokyo Institute of Technology where he received the M.Eng. He is now candidate for a D.Eng degree at the same department. His fields of interest include computer animation, motion capturing, and human behavior simulation.

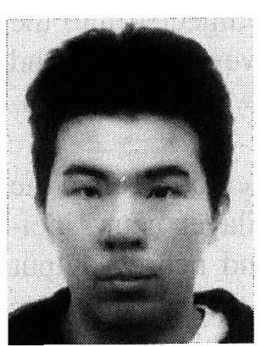

Ryota Kaihara was born in Okayama, Japan on December 9, 1984. He received the B.Eng degree from Tokyo Institute of Technology on March, 2007. He is now candidate for a M.Eng degree at Graduate School of Information Science and Engineering, Tokyo Institute of Technology. His fields of interest include computer animation, motion capturing, and human behavior simulation.

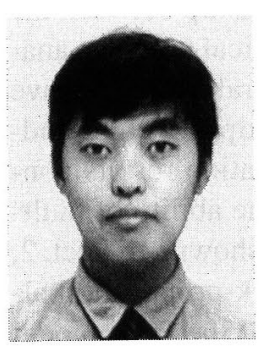

Suguru Saito received a B.Eng. degree in 1994 and a D.Eng. degree in 1999 from Tokyo Institute of Technology, Japan. Since 2004, he has been an associate professor at the department of computer science and engineering, Tokyo Institute of Technology, Japan. His fields of interest are image processing, image reconstruction, computer graphics, color science and human perception.

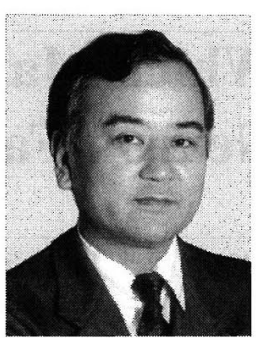

Masayuki Nakajima received the B.E.E. degree and Dr.Eng. degree from the Tokyo Institute of Technology, Japan, in 1969 and 1975, respectively. Since 1975 he had been with the department of imaging science and engineering, Tokyo Institute of Technology, Yokohama, Japan. He is now a professor at the department of computer science, the faculty of graduate school of information science and engineering, Tokyo Institute of Technology, Tokyo, Japan. His fields of interest are computer graphics, pattern recognition, image processing and virtual reality. 